\title{
The Application of Fractional Differential Equation to Mortgage Problems
}

\author{
Modebei M. I.*, Olaiya O. O. \\ Department of Mathematics Programme National Mathematical Centre, Abuja Nigeria \\ *Corresponding authorE-mail: modebeim@nmcabuja.org,gmarc.ify@gmail.com
}

\begin{abstract}
Suppose a customer takes out a fixed rate mortgage for $p$ Naira at an interest rate of $R \%$ per year, and wants to pay off the loan in $Z$ years. The immediate task is to find out what the annual payment should be so that the loan is indeed cleared in $Z$ years. In this work, we model this mortgage problem using difference equation and approximate the solution of the problem using a fractional differential equation. We thus make comparison between the solutions obtained from the conventional approach for calculating such debt and the fractional differential equation approach using a graph. We thereby make the conclusion that the use of fractional differential equation method enables a customer to pay off loans more quicker.
\end{abstract}

Keywords: Difference equation, fractional differential equation, mortgage problem, laplace transform, mittag-laffler function, gamma function.

\section{Introduction}

As old as normal, conventional, integer-order calculus. Born in 1695, In a letter correspondence, Marquis de L'Hospital asked Leibniz "What if the order of the derivative is $1 / 2$ ?" To this end, Leibniz replied in a prophetical way, "Thus it follows that will be equal to $x^{2} \sqrt[2]{2 d x: x)}$, an apparent paradox, from which one day useful consequences will be drawn." This letter of Leibniz was dated far back 30th September, 1695.[1].

In spite of its long history, fractional calculus was not considered eligible for any applications. This was due to its high complexity and lack of physical and geometric interpretation. Application of fractional calculus to real-world problems is only four decades old. Applications can be broadly categorized into: Modeling of Systems and Fractionalorder Control,[2].

Rigorous mathematical theory has been developed. Integer-order calculus is the special case. Geometrical interpretation or physical meaning exists. But not as straight forward as for the integer-order derivatives,[7].

A typical example of differential equations involving fractional derivatives. is the Bagley-Torvik equation of oscillatory processes with fractional damping[4]:

$$
\frac{d^{2}}{d x^{2}} f(x)+\alpha D_{t}^{\frac{3}{2}} f(x)+\beta f(x)=g(x)
$$

There are likewise both ODEs and PDEs. which has fractional derivatives that are Linear and non-linear. (Give reference) Existence and uniqueness of solutions have been established, analytical solutions are difficult to evaluate. Dedicated, elegant numerical methods exist as well,[6].

Some of the merit of fractional derivatives are [2]:

(1) Calculating time-fractional derivative of a function $f(t)$ at some $t=t_{1}$ requires all the past history, i.e. all $f(t)$ from $t=0$ to $t=t_{1}$.

(2) Fractional derivatives can be used for modeling systems with memory.

(3) Calculating space-fractional derivative of a function $f(x)$ at $x=x_{1}$ requires all non-local $f(x)$ values.

(4) Fractional derivatives can be used for modeling distributed parameter systems.

In this paper we shall see that one of such models formulated using difference equation can be accurately approximated using fractional differential equation. This fractional differential equation shall be solved using the approach of Laplace transform. 


\section{Preliminary notes}

\subsection{Gamma Function}

This is otherwise refered to at the generalization of the factorial for all real numbers, defined by

$$
\Gamma(x)=\int_{0}^{\infty} e^{-t} t^{x-1} d t \quad x \in R
$$

with the property that

$$
\begin{aligned}
& \Gamma(x+1)=x \Gamma(x) \\
& \Gamma(x)=(x-1) !
\end{aligned}
$$

\subsection{The Error Function}

This function is defined thus [10]

$$
\operatorname{Erf}(x)=\frac{2}{\sqrt{\pi}} \int_{0}^{x} e^{-t^{2}} d t
$$

the complementary error function $(E f r c)$ is a closely related function that can be written in terms of the Erf function as

$$
\operatorname{Erfc}(x)=1-\operatorname{Erf}(x)
$$

Notice closely that $\operatorname{Erf}(0)=0$ and $\operatorname{Erf}(\infty)=1$

\subsection{Mittag-Leffler function}

This function is a direct generalization of the conventional exponential function $e^{x}$, and it play a very important role in fractional calculus. The Mittag-Leffler function is defined in terms of power series as follows [10]

$$
\begin{aligned}
& E_{\alpha}(x)=\sum_{k=0}^{\infty} \frac{x^{k}}{\Gamma(\alpha k+1)}, \alpha>0 \quad \text { (one parameter } M-L \text { function) } \\
& E_{\alpha, \beta}(x)=\sum_{k=0}^{\infty} \frac{x^{k}}{\Gamma(\alpha k+\beta)}, \alpha>0 \quad \text { (two parameter } M-L \text { function) }
\end{aligned}
$$

It can be easily shown that

$$
\frac{d}{d x} E_{\alpha, \beta}(x)=\frac{1}{\alpha x}\left[E_{\alpha, \beta-1}(x)-(\beta-1) E_{\alpha, \beta}(x)\right]
$$

Note that $E_{\alpha, \beta}(0)=1$. Also, for some particular values of $\alpha$ and $\beta$, the Mittage-Leffler function reduces to some farmiliar functions. For example

$$
\begin{aligned}
& E_{1,1}(x)=e^{x} \\
& E_{1 / 2,1}(x)=e^{x^{2}} \operatorname{Erfc}(-x) \\
& E_{1,2}(x)=\frac{e^{x}-1}{x}
\end{aligned}
$$

\subsection{Riemann-Liouville Fractional Integral}

Definition 2.1:Let $v$ be a non negative number, $f$ be piecewise continuous on $I=(0, \infty)$ and integrable on any finite subinterval of $J=[0, \infty]$. Then for $t>0$ we define the Riemann-Liouville fractional integral of $f$ of order $v$ as

$$
{ }_{c} D_{x}^{-v} f(x)=\frac{1}{\Gamma(v)} \int_{c}^{x}(x-t)^{v-1} f(t) d t, \quad v>0
$$

This definition can be obtained in several ways but we shall consider the linear differential equation. We first state a lemma.

Lemma 2.2 (Replacement Lemma) Suppose that $f:[a, b] \rightarrow R$ is continuous, then

$$
\int_{a}^{x} \int_{a}^{x^{\prime}} f(t) d t d x^{\prime}=\int_{a}^{x}(x-t) f(t) d t, \quad x \in[a, b]
$$


Now consider the $n t h$ order linear differential equation with the initial conditions

$$
\left\{\begin{array}{l}
y^{(n)}=f(x) \\
y(c)=y^{\prime}(c)=y^{\prime \prime}(c)=\mathrm{L}=y^{(n-1)}(c)=0
\end{array}\right.
$$

Integrating $n$ times from $c$ to $x$ and from the second integration to the $n t h$ integration, we apply Lemma 2.2, we obtain

$$
y(x)=\int_{c}^{x} \frac{(x-t)^{n-1}}{(n-1) !} f(t) d t+\frac{(x-c)^{n-1}}{(n-1) !} y^{(n-2)}(c)+\mathrm{L}+(x-c) y^{\prime}(c)+y(c)
$$

since $y(c)=y^{\prime}(c)=y^{\prime \prime}(c)=\mathrm{L}=y^{(n-1)}(c)=0$ we get

$$
y(x)=\int_{c}^{x} \frac{(x-t)^{n-1}}{(n-1) !} f(t) d t
$$

We may rightly say that $y(t)$ is the $n t h$ integral of $f(x)$, thus;

$$
{ }_{c} D_{x}^{-v} f(x)=\frac{1}{(n-1) !} \int_{c}^{x}(x-t)^{n-1} f(t) d t
$$

Replacing $n$ with $v>0$ and recalling that $\Gamma(n)=(n-1)$ !, we therefore have

$$
{ }_{c} D_{x}^{-v} f(x)=\frac{1}{\Gamma(v)} \int_{c}^{x}(x-t)^{v-1} f(t) d t, \quad v>0
$$

\subsection{Riemann-Liouville fractional derivative}

The Riemann-Liouville fractional derivative can be defined using the definition of the fractional integral.

Definition 2 3: let $v=n-u$ where $0<v<1$ and $n$ is the smallest integer greater then $u$. The fractional derivative of $f(x)$ of order $u$ is

$$
D^{u} f(x)=D^{n}\left[D^{-v} f(x)\right]
$$

If we wish to find the fractional derivative of $f(x)=x^{\lambda}$ of order $v$, where $\lambda \geq 0$, in order to use the definition above, we interchange $u$ and $v$ and let $u=n-v$ where $0<u<1$. Then we have that $n=1$ and $u=1-v$, so that

$$
\begin{aligned}
D^{v} f(x) & =D^{1}\left[D^{-(1-v)} f(x)\right] \\
& =D^{1}\left[D^{-(1-v)} x^{\lambda}\right] \\
& =D^{1}\left[\frac{\Gamma(\lambda+1)}{\Gamma((\lambda-v+1)+1)} x^{\lambda-v+1}\right] \\
& =\frac{(\lambda-v+1) \Gamma(\lambda+1)}{(\lambda-v+1) \Gamma(\lambda-v+1)} x^{\lambda-v} \\
D^{v} f(x) & =\frac{\Gamma(\lambda+1)}{\Gamma(\lambda-v+1)} x^{\lambda-v}
\end{aligned}
$$

\section{Fractional differential equation and Laplace transform}

Here we shall apply Laplace transform to solve some fractional differential equation. This procedure ia analogous to solving the conventional differential equation with integer order using Laplace transform.

Definition 3.1 (Laplace Transform.): Let $f(x)$ be given for $t \geq 0$ and suppose that $f(x)$ is defined on some interval $I$. The Laplace transform of $f(t)$ denoted by $\mathrm{L}\{f(t)\}$ or equivalently $F(s)$ is defined by the equation

$$
\mathrm{L}\{f(t)\}=F(s)=\int_{0}^{\infty} e^{-s t} f(t) d t
$$

Theorem 3.2: Suppose that

(1) $f$ is piecewise continuous on the interval $0 \leq t \leq A$ for any positive $A$;

(ii) $|f(t)| \leq k e^{s t}$ where $t \geq M$, where $k, a, M$ are real constants and $k, M>0$. 
Then, $L\{f(t)\}=F(s)$ defined in (3) above exist for $s>a$.

Such functions are described as piecewise continuous, and of exponential order as $t \rightarrow \infty$.

$\mathrm{L}^{-1}\{f(t)\}$ is the inverse Laplace transform of $f(t)$ and is unique.

The Laplace transform is a Linear operator. that is closed under addition and scalar multiplication. The laplace transform of $t^{p}$ where $p>-1$ is given by

$$
\mathrm{L}\left\{t^{p}\right\}=\int_{0}^{\infty} e^{-s t} t^{p} d t=\frac{1}{s^{p+1}} \int_{0}^{\infty} e^{-x} x^{p} d x=\frac{\Gamma(p+1)}{s^{p+1}}
$$

and

$$
\mathrm{L}\left\{e^{\alpha t}\right\}=\int_{0}^{\infty} e^{(\alpha-s) t} d t=\frac{1}{s-\alpha}
$$

\subsection{Laplace transform of fractional integral}

The Laplace transform of $f(t)$ of order $v$ is given as

$$
\mathrm{L}\left\{D^{-v} f(t)\right\}=\frac{1}{\Gamma(v)} \mathrm{L}\left\{t^{v-1}\right\} \mathrm{L}\{f(t)\}=s^{-v} F(s)
$$

Which is the Laplace transform of (1) above.

\subsection{Laplace transform of fractional derivative}

The Laplace transform of integer order operator of $f^{(n)}$ is given as

$$
\begin{aligned}
\mathrm{L}\left\{f^{(n)}(t)\right\} & =s^{n} F(s)-s^{n-1} f(0)-s^{n-2} f^{\prime}(0)-\Lambda-f^{(n-1)}(0) \\
& =s^{n} F(s)-\sum_{k=0}^{n-1} s^{n-k-1} f^{(k)}(0)
\end{aligned}
$$

we thus recall from (2) and noting that $u=n-v$, then

$$
D^{v} f(t)=D^{n}\left[D^{-(n-v)} f(t)\right]
$$

Assume that $\mathrm{L}\{f(t)\}$ exist, then

$$
\begin{aligned}
\mathrm{L}\left\{D^{v} f(t)\right\} & =\mathrm{L}\left\{D^{n}\left[D^{-(n-v)} f(t)\right]\right\} \\
& =s^{n} \mathrm{~L}\left\{D^{n}\left[D^{-(n-v)} f(t)\right]\right\}-\left.\sum_{k=0}^{n-1} s^{n-k-1} D^{k}\left[D^{-(n-v)} f(t)\right]\right|_{t=0} \\
& =s^{n}\left[s^{-(n-v)} F(s)\right]-\sum_{k=0}^{n-1} s^{n-k-1} D^{k-(n-v)} f(0) \\
& =s^{v} F(s)-\sum_{k=0}^{n-1} s^{n-k-1} D^{k-(n-v)} f(0)
\end{aligned}
$$

More precisely. if we take $n=2$ we obtain

$$
\mathrm{L}\left\{D^{v} f(t)\right\}=s^{v} F(s)-s D^{-(2-v)} f(0)-D^{-(1-v)} f(0), \quad 0<v \leq 2
$$

To illustrate this we thus solve the fractional differential equation $D^{1 / 2} f(t)=\alpha f(t)$.

Finding the Laplace transform of both sides we obtain

$$
s^{1 / 2} F(s)-D^{-1 / 2} f(0)=\alpha F(s)
$$

It is quite easy to see that $D^{-1 / 2} f(0)$ is a constant say $\lambda$, so that we have

$$
\begin{aligned}
& s^{1 / 2} F(s)-\lambda=\alpha F(s) \\
& F(s)=\frac{\lambda}{s^{1 / 2}-\alpha}
\end{aligned}
$$

Finding the inverse Laplace transform we finally obtain

$$
f(t)=\mathrm{L}^{-1}\left\{\frac{\lambda}{s^{1 / 2}-\alpha}\right\}=\lambda t^{-1 / 2} E_{1 / 2,1 / 2}\left(\alpha t^{1 / 2}\right)
$$




\section{Main results}

Suppose a customer decides to obtain a fixed rate mortgage for $p$ Naira at an interest rate of $R \%$ per year ( $r \%$ per month), and wants to pay off the loan in $Z$ years (in $z \%$ months). In this work we shall find out what the yearly payment should be so that the loan is cleared in $Z$ years, $[8,9]$. Suppose now that the yearly payment is $A$ Naira and the balance of debt at the end of period $t$ years is $f(t)$ where $t \neq Z$. At the instance when $t$ and $Z$ coincides we seek the balance of debt $f(Z)$ to be identically zero, i.e all debt paid off. So when $P$ and $R$ are given, we have that the yearly payment will be

$$
A:=\frac{P R(1+R)^{Z}}{(1+R)^{Z}-1}
$$

we shall consider monthly payments in lieu of yearly payments. Thus, the balance of debt $f(t)$ is thus calculated from the ivp given as;

$$
f^{\prime}(t)=r f(t)-A
$$

with

$$
f(0)=p .
$$

Solving (6), we get

$$
f(t):=p e^{r t}-\frac{A}{r}\left[e^{Z}-1\right],
$$

Intuitively, $e^{r t} \approx(1+r)^{z}$, where we now assume that $t \approx z$ for a total debt pay off so that we may write (7) as

$$
f(t):=p(1+r)^{z}-\frac{A}{r}\left[(1+r)^{z}-1\right]
$$

If the change happens incrementally rather than continuously then differential equations have their shortcomings. Instead we will use difference equations which are recursively defined sequences.

Here, we shall consider the discrete case for this model given by the governing difference equation

$$
f_{n+1}=r f_{n}-A
$$

where $f_{0}=p$. Solving (9), by setting $n=0,1,2, \mathrm{~K}, n$, where $n=z$ in this case, we obtain the solution,

$$
f_{n}=r^{n} p-A \frac{\left(1-r^{n}\right)}{1-r}
$$

I can be easily shown that for $n>0$ the result obtained is not in any way realistic, hence the solution (10) is not a good approximation of (9).

Now, for a better approximation of the discrete case as in (9) we can model the problem (6) in fractional differential equation as

$$
D^{v} f(t)=r f(t)-A, \quad 0<v<1
$$

thereby approximating (8).

We are thus faced with solving (11) using Laplace transform. Taking the Laplace transform of both sides of (11) that is $\mathrm{L}\left\{D^{v} f(t)\right\}=r \mathrm{~L}\{f(t)\}-\mathrm{L}\{A\}$

we then have

$$
s^{v} F(s)-D^{-(1-v)} f(0)=r F(s)-\frac{A}{s}
$$

We thus take $D^{-(1-v)} f(0)=\lambda$, where $\lambda$ is a constant. Then

$$
\begin{aligned}
& s^{v} F(s)-\lambda=r F(s)-\frac{A}{s} \\
& F(s)=\frac{\lambda s-A}{s\left(s^{v}-r\right)} \\
&=\frac{\lambda}{s^{v}-r}-\frac{A}{s\left(s^{v}-r\right)}
\end{aligned}
$$

Finding the inverse Laplace transform yields

$$
f_{v}(t)=\lambda t^{v-1} E_{v, v}\left(r t^{v}\right)-A t^{v} E_{v, v+1}\left(r t^{v}\right)
$$

It is easy to see that 
and

$$
\lim _{t \rightarrow 0^{+}} t^{v-1} E_{v, v}\left(r t^{v}\right)=1
$$

$$
\lim _{t \rightarrow 0^{+}} t^{v} E_{v, v+1}\left(r t^{v}\right)=0
$$

so that we may conclude that $\lambda$ to be the amount loaned, that is, the initial debt whose value is $p$. Then (12) becomes

$$
f_{v}(t)=p t^{v-1} E_{v, v}\left(r t^{v}\right)-A t^{v} E_{v, v+1}\left(r t^{v}\right)
$$

\subsection{Numerical example}

Mr Mark needs to buy a new house. he takes a 5-years loan of N2.5 million at a fixed interest rate of 8\%, compounded monthly. We are now faced with calculating Mr Mark's remaining debt after t months and comparing the result using (8) and (13).

Note that:

$$
\begin{aligned}
& z=5(12)=60 \\
& P=2500000 \\
& r=0.08 / 12=0.0067 \\
& A=\frac{2500000(0.0067)(1+0.0067)^{60}}{(1+0.0067)^{60}-1}=50738.9
\end{aligned}
$$

Now plugging all these values into (8) and (13) we have

$$
f(t):=2500000(1+0.0067)^{t}-\frac{50738.9}{0.0067}\left[(1+0.0067)^{t}-1\right]
$$

and

$$
f_{v}(t)=2500000 t^{v-1} E_{v, v}\left(0.0067 t^{v}\right)-50738.9 t^{v} E_{v, v+1}\left(0.0067 t^{v}\right)
$$

Table 1 (Here the $-v e$ indicates surplus payment after the debt has been paid off completely.)

\begin{tabular}{ccccccc}
\hline$t$ (months) & $f(t)$ & $f_{0.99}(t)$ & $f_{0.98}(t)$ & $f_{0.96}(t)$ & $f_{0.94}(t)$ & $f_{0.92}(t)$ \\
\hline 0 & $2.500 \times 10^{6}$ & Indeterminate & Indeterminate & Indeterminate & Indeterminate & Indeterminate \\
48 & $5.832 \times 10^{5}$ & $5.091 \times 10^{5}$ & $4.472 \times 10^{5}$ & $3.363 \times 10^{5}$ & $2.408 \times 10^{5}$ & $1.589 \times 10^{5}$ \\
50 & $4.892 \times 10^{5}$ & $4.168 \times 10^{5}$ & $3.57 \times 10^{5}$ & $2.505 \times 10^{5}$ & $1.595 \times 10^{5}$ & $8.221 \times 10^{4}$ \\
52 & $3.939 \times 10^{5}$ & $3.233 \times 10^{5}$ & $2.658 \times 10^{5}$ & $1.639 \times 10^{5}$ & $7.774 \times 10^{4}$ & $5.208 \times 10^{3}$ \\
54 & $2.974 \times 10^{5}$ & $2.287 \times 10^{5}$ & $1.735 \times 10^{5}$ & $7.66 \times 10^{4}$ & $-4.592 \times 10^{3}$ & $-7.213 \times 10^{4}$ \\
56 & $1.996 \times 10^{5}$ & $1.329 \times 10^{5}$ & $8.028 \times 10^{4}$ & $-1.15 \times 10^{4}$ & $-8.748 \times 10^{4}$ & $-1.498 \times 10^{5}$ \\
58 & $1.005 \times 10^{5}$ & $3.589 \times 10^{4}$ & $-1.403 \times 10^{4}$ & $-1.004 \times 10^{5}$ & $-1.709 \times 10^{5}$ & $-2.279 \times 10^{5}$ \\
60 & -3.113 & $-6.229 \times 10^{4}$ & $-1.094 \times 10^{5}$ & $-1.901 \times 10^{5}$ & $-2.55 \times 10^{5}$ & $-3.064 \times 10^{5}$ \\
\hline
\end{tabular}

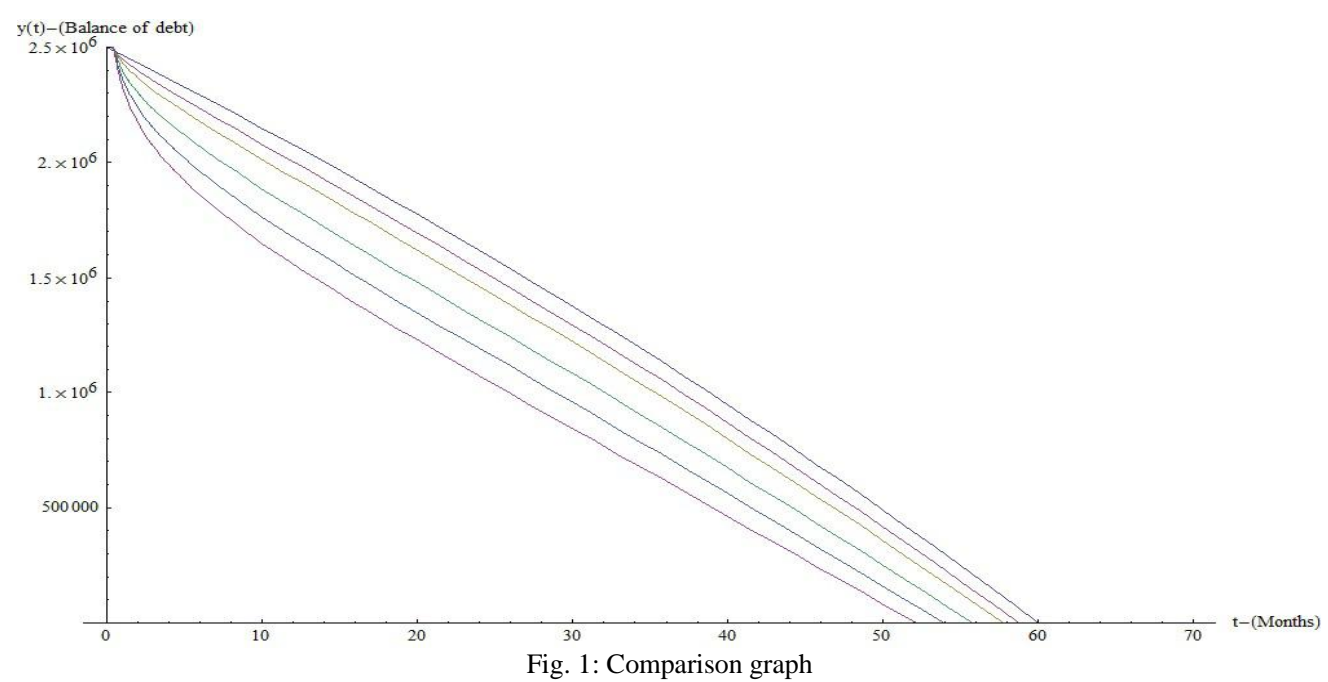


In Fig. 1, the upper-most curve is $f(t)$ in (14), the next curves after it are $f_{v}(t)$ in (15), for $v=0.99,0.98,0.96,0.94,0.92$ respectively. We thus deduce that as $v \rightarrow 0$ the customer would off-set the loan much quicker if any of the approximating (fractional) models was used. In fact, it appears that the smaller the value of $v$, the sooner Mr Mark pays off the loan.

\section{Conclusion}

Apparently, fractional differential equation approximates the model formulated by difference equation more efficiently and accurately. In the study of fractional differential equation we can observe that a lot of concepts must be known. this is what makes this type of differential equation more complex than the conventional integer-order differential equation (ordinary and partial). Researches are still on going in this direction.

\section{References}

[1] Ross, B. (Editor): Fractional Calculus and its Applications, Lecture Notes in Mathematics no. 457, Springer Verlag, Berlin 1975. [Proc. Int. Conf. held at Univ. of New Haven, USA, 1974].

[2] Samko S.G., Kilbas, A.A. and O.I. Marichev: Fractional Integrals and Derivatives, Theory and Applications, Gordon and Breach, Amsterdam 1993. [Engl. Transl. from Russian, Integrals and Derivatives of Fractional Order and Some of Their Applications.

[3] Miller, K.S. and B. Ross: An Introduction to the Fractional Calculus and Fractional Differential Equations, Wiley, New York 1993.

[4] Beyer, H. and S. Kempfle: Definition of physically consistent damping laws with fractional derivatives, ZAMM, 75 (1995), 623-635.

[5] Nonnenmacher, T.F. and R. Metzler: On the Riemann-Liouville fractional calculus and some recent applications, Fractals, 3 (1995), 557-566.

[6] Matignon, D.: Stability results for fractional differential equations with applications to control processing, Proceedings Computational Engineering in Systems and Application multiconference, IMACS, IEEE-SMC, Lille, France, July 1966, pp. 963-968.

[7] Barret, J. H.: Differential equations of non-integer order, Canad. J. Math., 6 (1954), 529-541.

[8] Jak a Cvitani'c and Fernando Zapatero: Introduction to the Economics and Mathematics of Financial Markets MIT Press Cambridge, Massachusetts London, HG106.C86 2004.

[9] Kimeu, Joseph M., "Fractional Calculus: Definitions and Applications." Masters Theses \& Specialist Projects. Paper 115. http://digitalcommons.wku.edu/theses/115 (2009).

[10] Shah P.V. and jana R. K.: Results on Generalized Mittag-Leffler Function via Laplace Transform. Applied.

[11] Mathematical Sciences, Vol. 7, no. 12, 567 - 570, 2013 Seybold, H., Hilfer, R.: Numerical algorithm for calculating the generalized MittagLeffler function, SIAM J. Numer. Anal. 47(1), 69-88 (2008/09). 